\title{
Clinical implications of the dual blockade of the PD-1/PD-L1 and vascular endothelial growth factor axes in the treatment of hepatocellular carcinoma
}

\author{
Naoshi Nishida \\ Department of Gastroenterology and Hepatology, Kindai University Faculty of Medicine, Osaka, Japan \\ Correspondence to: Naoshi Nishida, MD, PhD. Department of Gastroenterology and Hepatology, Kindai University Faculty of Medicine, 377-2 \\ Ohno-Higashi Osaka-Sayama, Osaka 589-8511, Japan. Email: naoshi@med.kindai.ac.jp. \\ Comment on: Shigeta K, Datta M, Hato T, et al. Dual Programmed Death Receptor-1 and Vascular Endothelial Growth Factor Receptor-2 Blockade \\ Promotes Vascular Normalization and Enhances Antitumor Immune Responses in Hepatocellular Carcinoma. Hepatology 2020;7 1:1247-61.
}

Submitted Oct 05, 2019. Accepted for publication Oct 16, 2019.

doi: $10.21037 /$ hbsn.2019.10.18

View this article at: http://dx.doi.org/10.21037/hbsn.2019.10.18

Currently, liver cancer is the fourth most common cause of cancer mortality worldwide. The overwhelming majority of liver cancers are hepatocellular carcinomas (HCC), developing on the background of chronic liver diseases, including chronic hepatitis $\mathrm{B}$, chronic hepatitis $\mathrm{C}$, and non-alcoholic steatohepatitis (1). Despite the recent progress in the treatment of HCC, it remains largely refractory, with a high recurrence rate even after therapeutic intervention (2). Numerous agents, including tyrosine-kinase inhibitors (TKIs), antibodies blocking the vascular endothelial growth factor (VEGF)/ VEGF receptor (VEGFR) axis, and immune checkpoint inhibitors, are now available for the treatment of advanced stage HCC (3). However, their antitumor responses are still unsatisfactory.

Although HCC is a known hypervascular tumor, with oxygen and nutrition supplied by tumor vessels, trials using the anti-vascular endothelial growth factor-A (VEGF-A) antibody failed to demonstrate enough clinical benefits in HCC treatment (4). In addition, recent phase III clinical trials on immune checkpoint monotherapy failed to reveal its superiority in the overall survival of the active treatment group compared to the control group, although durable responses were observed in some cases. Therefore, from a clinical point of view, there exists much scope for the improvement and development of systemic therapy in HCC using anti-VEGF and anti-programmed cell death-1 (PD-1) antibodies (4-6). Recently, a combination of immune checkpoint inhibitors and anti-angiogenic agents was reported to cause a considerable reduction in tumor burden in human HCC patients $(3,7)$, suggesting that a synergistic effect could be expected from the dual blockade of these two pathways in the treatment of human HCC; several phase III clinical trials for this type of combination are now ongoing.

\section{Hypoxia, VEGF, and immune suppressive status of HCC}

Theoretically, treatment with anti-angiogenic agents could induce starvation in HCC cells, attributed to a lack of blood supply. However, as described above, monotherapy with anti-VEGF-A antibody failed to yield a sufficient antitumor effect in human HCC patients. In contrast, recent studies reported that anti-angiogenesis could induce normalization of tumor vessel architecture (8), leading to an improvement in the immunosuppressive microenvironment of tumor.

In HCC, tissue hypoxia induces VEGF and serum VEGF levels are associated with vascularity and aggressive behavior of the tumor (9). The VEGF signal stimulates neovascularization where leaky nascent vessels with loose pericyte coverage appear (Figure 1). In this setting, high interstitial fluid pressure could inhibit the infiltration of immune cells into tumor tissues. Lack of adhesion molecules on the endothelial cells of nascent tumor vessels could also impair the extravasation of $\mathrm{T}$ cells (10). Therefore, blockade of tumor neovascularization by anti-angiogenic agents could normalize the leaky vascular network and promote 


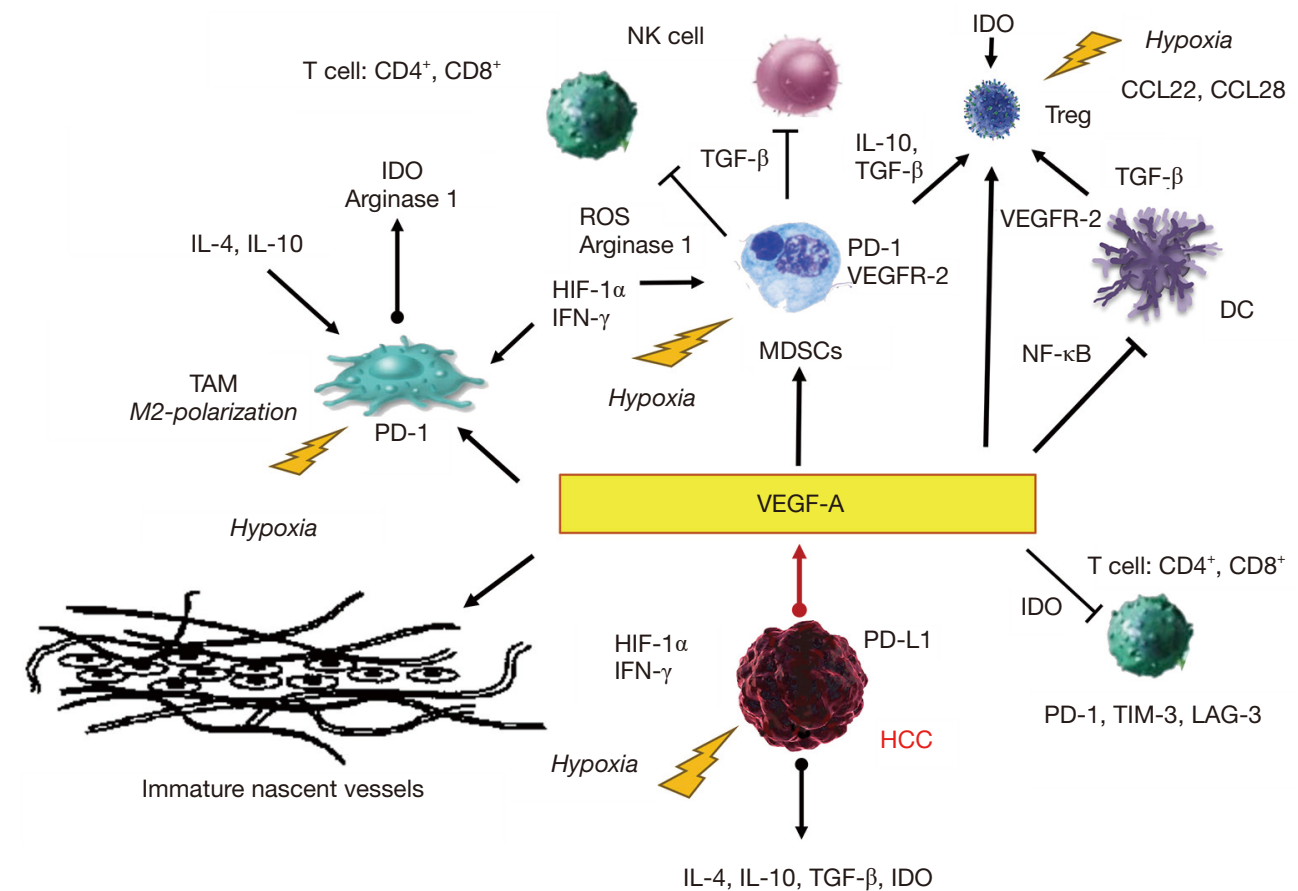

Figure 1 Involvement of cellular and humoral components of immunosuppressive microenvironment of HCC under hypoxia and VEGF. Mutual interactions between cellular components and humoral mediators, and induction of the immune suppressive molecules under hypoxia are shown. The oxygen supply from blood of tumor neo-vasculatures generally, can not compensate the increased oxygen consumption of tumors; pruning of tumor vessel aggravates tumor hypoxia. Hypoxia and subsequent induction of VEGF-A play a central role for establishment of immunosuppressive microenvironment of HCC. The arrows indicate the promotion of activation and the T-bar arrow head shows inhibitor of each immune cell components, respectively. CCL22 and 28, chemokine motif ligand-22 and -28; DC, dendric cell; HIF-1 $\alpha$, hypoxia-inducible factor-1 $\alpha$; IDO, indoleamine 2,3-dioxygenase; LAG-3, lymphocyte activating gene 3; MDSC, myeloidderived suppressor cells; TAM, tumor-associated macrophage; TIM-3, T-cell immunoglobulin and mucin domain 3; Treg, regulatory T cell; ROS, reactive oxygen species.

the induction of immune cells into tumors.

Moreover, the abnormal tumor neovasculature blood supply cannot compensate for the increased oxygen consumption in tumors. Tumor vessel pruning aggravates hypoxia in the tumor and leads to the expression of immune check point molecules in the myeloid-derived suppressor cells (MDSCs) and macrophages through the action of hypoxia-inducible factor- $1 \alpha(11,12)$. Furthermore, hypoxia could recruit regulatory $\mathrm{T}$ cells (Treg) through the upregulation of chemokine motif (C-C motif) ligand-22 and -28 and induce the polarization of tumorassociated macrophages to M2-like phenotype. Elevated VEGF inhibits the maturation of dendric cells (DCs) by activating $\mathrm{NF}-\kappa \mathrm{B}$ and promotes the production of indoleamine 2, 3-dioxygenase (IDO) that inhibits T-cell activation and proliferation and the induction of Treg $(12,13)$. VEGF also mediates $\mathrm{CD} 8^{+} \mathrm{T}$ cell exhaustion by inducing the expression of immune suppressive receptors, such as PD-1, lymphocyte activating gene 3, and T-cell immunoglobulin and mucin domain 3. Furthermore, high levels of VEGF induce the expression of Fas ligand (FasL) on tumor endodermal cells, eliminating $\mathrm{CD}^{+} \mathrm{T}$ cells and reducing their cytotoxic activity. It also promotes the accumulation of MDSCs that impair $\mathrm{CD}^{+}$and $\mathrm{CD} 8^{+}$ T-cell responses and inhibits the activity of natural killer cells via binding of TGF- $\beta$ (Figure 1).

Concurrent hypoxia, caused by immature neovasculature, induces several humoral factors that could play a significant role in the establishment of immune suppressive microenvironment, including PD-L1, IDO, interleukin (IL)-4, and IL-10 (12,13). As hypoxia and neovascularization of the tumor could construct the immune suppressive status of HCC, it is reasonable to speculate that the appropriate dose of anti-angiogenic agents could subdue immune suppressive 


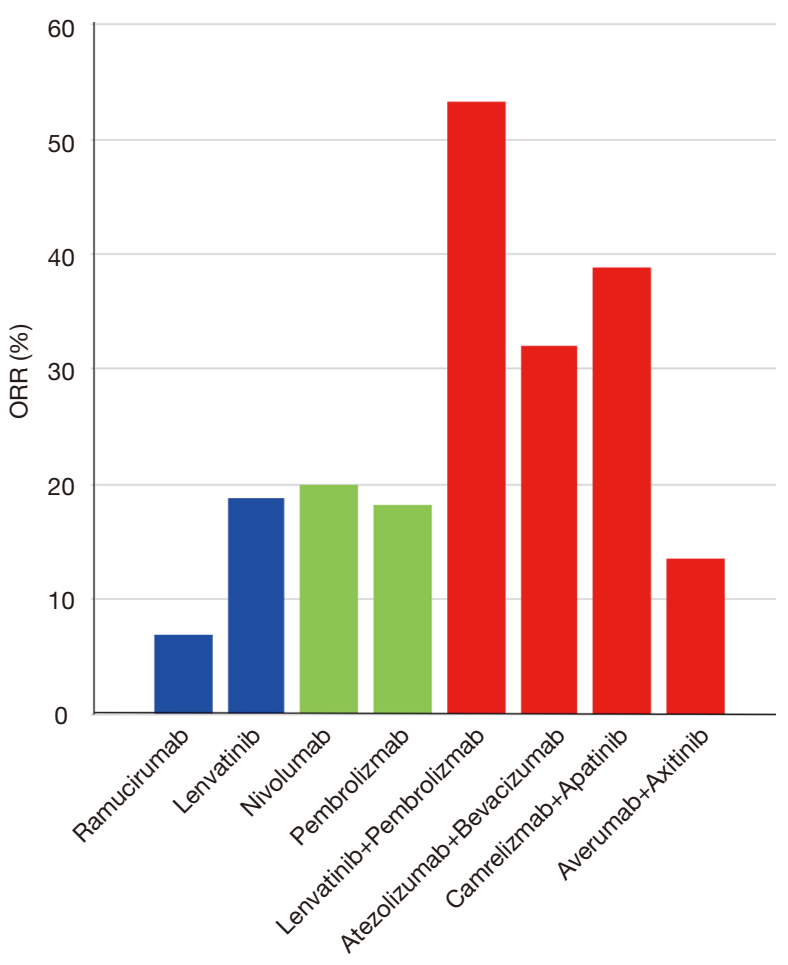

Figure 2 ORRs of anti-angiogenesis agent, anti-PD-1/PD-L1 monotherapies, and combination therapies. The blue bar indicates ORRs of anti-angiogenesis agent monotherapy. Similarly, the green bar shows ORRs of anti-PD-1/PD-L1 monotherapies, and the red bar shows those of combination therapies, respectively. ORR, objective response rate.

hormonal and cellular components and enhance the effect of immune checkpoint inhibitors (Figure 1).

In accordance, Shigeta $e t$ al. reported the synergistic antitumor effect observed with the combination of anti-PD-1 and anti-VEGFR-2 antibodies in HCC, using orthotopic murine models (14). Although anti-PD-1 monotherapy shows a minor survival benefit in a mouse model and antiVEGFR-2 monotherapy fails to demonstrate a survival advantage, the combination of these two antibodies significantly inhibits tumor growth and extends survival. Combination therapy with these agents triggered an increase in cytotoxic $\mathrm{CD}^{+} \mathrm{T}$ cells and the M1/M2 ratio of TAM and a reduction in Treg and CCR2+ monocyte infiltration. These findings suggested that blockade of the VEGF signal could reverse the immunosuppressive microenvironment in the mouse HCC model through the reduction of immune suppressive molecules. Anti-angiogenesis induces the expression of PD-L1 on HCC through the secretion of interferon- $\gamma$, after blockade of VEGFR-2 on endothelial cells. Conversely, combination therapy increases PD-1 expression on $\mathrm{CD}^{+}$cells that play an important role in the normalization of vascular formation. Collectively, anti-PD-1 and anti-VEGF treatment could establish a complementary role in the reprogramming of the immunosuppressive microenvironment and normalization of vasculature in HCC.

\section{Combination of dual blockade of the VEGF/ VEGFR and PD-1/PD-L1 axes in human HCC cases}

As described above, several clinical trials combining immune checkpoint inhibitors and anti-angiogenic agents are ongoing in HCC patients. So far, the combination of atezolizumab (an anti-PD-L1 antibody) and bevacizumab (an anti-VEGF-A antibody) achieved the objective response rate (ORR) of $32 \%$ in advanced HCC cases. Similarly, the combinations of camrelizumab (an anti-PD-1 antibody) and apatinib (a VEGFR-2-TKI), and averumab (an anti-PD-L1 antibody) and axitinib (a multikinase inhibitor that mainly inhibits VEGFR-tyrosine kinase) reportedly demonstrated ORRs of $38.9 \%$ and $13.6 \%$, respectably (Figure 2) (15). Several phase III clinical trials of combination therapy with dual blockade are currently ongoing for advanced stage of HCC patients. In addition, combination with an anti-PD-L1 antibody, pembrolizumab, and a multikinase inhibitor, lenvatinib, also achieved a high ORR of $53.3 \%$ (15); lenvatinib shows a strong inhibitory effect on VEGFR-tyrosine kinase (Figure 2).

The antitumor responses in the HCC murine model reported by Shigeta et al. are consistent with the observations in clinical studies (14). Collectively, immune checkpoint inhibitors and anti-angiogenic agents can be key agents and present a promising combination for systemic therapy of human HCC.

\section{Acknowledgments}

Funding: None.

\section{Footnote}

Provenance and Peer Review: This article was commissioned by the editorial office, Hepatobiliary Surgery and Nutrition. The article did not undergo external peer review.

Conflicts of Interest: The author has completed the ICMJE uniform disclosure form (available at http://dx.doi. 
org/10.21037/hbsn.2019.10.18). The author has no conflicts of interest to declare.

Ethical Statement: The author is accountable for all aspects of the work in ensuring that questions related to the accuracy or integrity of any part of the work are appropriately investigated and resolved.

Open Access Statement: This is an Open Access article distributed in accordance with the Creative Commons Attribution-NonCommercial-NoDerivs 4.0 International License (CC BY-NC-ND 4.0), which permits the noncommercial replication and distribution of the article with the strict proviso that no changes or edits are made and the original work is properly cited (including links to both the formal publication through the relevant DOI and the license). See: https://creativecommons.org/licenses/by-nc-nd/4.0/.

\section{References}

1. Yang JD, Hainaut P, Gores GJ, et al. A global view of hepatocellular carcinoma: trends, risk, prevention and management. Nat Rev Gastroenterol Hepatol 2019;16:589-604.

2. Villanueva A. Hepatocellular Carcinoma. N Engl J Med 2019;380:1450-62.

3. Yarchoan M, Agarwal P, Villanueva A, et al. Recent Developments and Therapeutic Strategies against Hepatocellular Carcinoma. Cancer Res 2019;79:4326-30.

4. Siegel AB, Cohen EI, Ocean A, et al. Phase II trial evaluating the clinical and biologic effects of bevacizumab in unresectable hepatocellular carcinoma. J Clin Oncol 2008;26:2992-8.

5. Voutsadakis IA. PD-1 inhibitors monotherapy in hepatocellular carcinoma: Meta-analysis and systematic

Cite this article as: Nishida N. Clinical implications of the dual blockade of the PD-1/PD-L1 and vascular endothelial growth factor axes in the treatment of hepatocellular carcinoma. HepatoBiliary Surg Nutr 2020;9(5):640-643. doi: 10.21037/ hbsn.2019.10.18 review. Hepatobiliary Pancreat Dis Int 2019;18:505-10.

6. Scheiner B, Kirstein MM, Hucke F, et al. Programmed cell death protein-1 (PD-1)-targeted immunotherapy in advanced hepatocellular carcinoma: efficacy and safety data from an international multicentre real-world cohort. Aliment Pharmacol Ther 2019;49:1323-33.

7. Nishida N, Kudo M. Immune checkpoint blockade for the treatment of human hepatocellular carcinoma. Hepatol Res 2018;48:622-34.

8. Jain RK. Normalization of tumor vasculature: an emerging concept in antiangiogenic therapy. Science 2005;307:58-62.

9. Llovet JM, Pena CE, Lathia CD, et al. Plasma biomarkers as predictors of outcome in patients with advanced hepatocellular carcinoma. Clin Cancer Res 2012;18:2290-300.

10. Teng MW, Ngiow SF, Ribas A, et al. Classifying Cancers Based on T-cell Infiltration and PD-L1. Cancer Res 2015;75:2139-45.

11. Grunewald M, Avraham I, Dor Y, et al. VEGF-induced adult neovascularization: recruitment, retention, and role of accessory cells. Cell 2006;124:175-89.

12. Prieto J, Melero I, Sangro B. Immunological landscape and immunotherapy of hepatocellular carcinoma. Nat Rev Gastroenterol Hepatol 2015;12:681-700.

13. Yi M, Jiao D, Qin S, et al. Synergistic effect of immune checkpoint blockade and anti-angiogenesis in cancer treatment. Mol Cancer 2019;18:60.

14. Shigeta K, Datta M, Hato T, et al. Dual Programmed Death Receptor-1 and Vascular Endothelial Growth Factor Receptor-2 Blockade Promotes Vascular Normalization and Enhances Antitumor Immune Responses in Hepatocellular Carcinoma. Hepatology 2020;71:1247-61.

15. Kudo M. Combination Cancer Immunotherapy with Molecular Targeted Agents/Anti-CTLA-4 Antibody for Hepatocellular Carcinoma. Liver Cancer 2019;8:1-11. 